\title{
KINEMATICS AND WARP IN NGC 456.5
}

\author{
S. Casertano ${ }^{1}$. R. Sancisi ${ }^{2}$ and T. S. van Albada ${ }^{2}$ \\ 1 Institute for Advanced Study, Princeton, NJ 08540 - USA \\ 2 Kapteyn Laboratorium. Postbus 800, 9700 AV Groningen - The Netherlands
}

We present preliminary results of the analysis of WSRT observations of the edge-on spiral NGC 4565 (Sancisi 1985). The neutral hydrogen layer of this galaxy extends to $1.3 \mathrm{R}_{25}$, permitting to trace the rotation curve outside the optical edge of the system, and is warped in the outer parts.

The rotation curve is obtained by constructing model velocity profiles at different positions along the major axis, and matching them to the observed profiles. The uncertainties in the derived velocities are of about $5 \mathrm{~km} / \mathrm{s}$. The relevant features of the rotation curve are a steep inner maximum and a steady slow decline of the rotation velocity in the outer parts.

In the inner parts, the rotation velocity quickly rises to reach a maximum value of about $260 \mathrm{~km} / \mathrm{s}$ at about $5 \mathrm{kpc}$ from the center. After the inner maximum, the velocity shows a sharp, faster-than-keplerian drop of about $30 \mathrm{~km} / \mathrm{s}$. If real, this feature would imply a nearly homogeneous, highly flattened (axis ratio $<0.5$ ) nuclear mass component, comprising at least $20 \%$ of the disk mass within one disk scale length. These characteristics do not agree with the properties of the observed bulge. Alternatively, the inner maximum may be only apparent, due to either non-circular motions or irregularities in the $\mathrm{HI}$ distribution.

At larger radii the rotation velocity exhibits a broad maximum ( $v=250 \mathrm{~km} / \mathrm{s}$ at $17 \mathrm{kpc}$ ). past which it slowly declines to about $225 \mathrm{~km} / \mathrm{s}$. The amount of the velocity drop implies that a good fraction of the total mass within the last measured point is probably in a non-luminous form.

The disk of NGC 4565 shows a warp both in the HI and in the optical image (van der Kruit and Searle 1981). At first sight, the warps in the two components do not coincide, in that the plane of the gas appears to become tilted before the stars do. However, a detailed model of the warp. made possible by the high resolution and quality of the maps, shows that the difference can be entirely due to projection effects. The model successfully reproduces the observed height above the galactic plane on both sides of the galaxy with a unique tilted-ring model. The tilt is rather abrupt, with the inclination increasing from 0 to 10 degrees in less than one disk scalelength, and then probably leveling off.

A more detailed description of our analysis is in preparation.

\section{REFERENCES}

Sancisi 1985, in preparation.

van der Kruit, P. C. and Searle, L. 1981, Astr. Ap., 95, 105. 